\title{
SUSCEPTIBILIDADE DO TRIATOMA INFESTANS A DIFERENTES CEPAS DE TRYPANOSOMA CRUZI ISOLADAS DE PACIENTES CHAGÁSICOS DO TRIÂNGULO MINEIRO
}

\author{
Roberta Aparecida Nirschl, José Maria Soares Júnior, Eliane Maria Pirani, \\ José Umberto Franciscon e Luis Eduardo Ramírez
}

\begin{abstract}
No presente trabalho avaliou-se a susceptibilidade do Triatoma infestans a sete cepas de Trypanosoma cruzi, isoladas e adaptadas em camundongos, provenientes de pacientes chagásicos do Triângulo Mineiro. Alimentou-se umtotal de 200 triatomíneos sobre camundongos infectados, na fase aguda, com as diferentes cepas e a cada 30 dias durante 5 meses, realizouse as leituras pelosmétodos da compressão e dejeção espontânea. A positividade do $\mathrm{T}$. infestans frente as diferentes cepas foi de 62,08\% acompanhado de uma elevada mortalidade (60\%). Conclui-se que o $\mathrm{T}$. infestans é pouco susceptivel as cepas isoladas, fato que pode explicar a baixa postividade dos xenodiagnósticos realizados em pacientes chagásicos crônicos do Triângulo Mineiro.
\end{abstract}

Palavras-chaves: Susceptibilidade do Triatoma infestans. Trypanosoma cruzi. Xenodiagnóstico.

Triatomainfestanséa espécie vetora domiciliada de maior distribuição geográfica da América do Sul, habitando uma ampla área, desde os áridos planaltos no Peru e planícies temperadas da Argentina até as zonas tropicais secas do nordeste brasileiro. Atualmente, limita-se a ocupar habitats humanos artificiais na maior parte da sua zona de distribuição. Oitenta e quatro a noventa e nove porcento da alimentação da espécie vem do sangue dos seres vivos existentes na zona de domiciliação, principalmente, homens, cães, gatos e roedores ${ }^{8}$. É considerada a principal espécie vetora nas regiões centro-oeste e sudeste do Brasil ${ }^{6}$ e segundo Camargo e Takeda ${ }^{5}$, tem sido a espécie preferida para a realização do xenodiagnótico devido a sua importância epidemiológica, porém, tem sido questionado se as espécies vetoras locais das áreas endêmicas são mais facilmente infectadas com as cepas locais do parasita que as não naturais da região ${ }^{210}$.

Graus variáveis de susceptibilidade tem sido encontrados entre as diversas espécies de tritomíneos frente às diferentes cepas do $T$. cruzi ${ }^{3}{ }^{9}$. Essa susceptibilidade pode estar relacionada a fatores

\footnotetext{
Departamento de Ciências Biológicas, Disciplina de Parasitologia da Faculdade de Medicina do Triângulo Mineiro, Uberaba, MG.

Endereço para correspondência: Prof. Luis Eduardo Ramírez. Pça. Thomaz Uhoa s/n, 38025-440, Uberaba, MG, Brasil. Recebido para publicação em 14/04/94.
}

nutricionais, estímulos fisiológicos apropriados ou à presença de substâncias inibitórias no trato intestinal do inseto que poderiam intervir no desenvolvimento do parasita ${ }^{15}$, ou poderiam estar sob controle genético ${ }^{7}$.

Este trabalho pretende avaliar a susceptibilidade do Triatoma infestans a diferentes cepas de Trypanosoma cruzi, isoladas de pacientes chagásicos do Triângulo Mineiroe inoculados em camundongos e em processo de adaptação a esse novo hospedeiro.

\section{MATERIAL E MÉTODOS}

Utilizaram-se sete cepas de Trypanosoma cruzi provenientes de pacientes chagásicos crônicos do Triângulo Mineiro, denominadas: A, B, E, G, H, $\mathrm{R}$ e W. Essas cepas foram isoladas através do xenodiagnóstico e inoculadas em camundongos não isogênicos para estudos futuros.

Empregaram-se de 20 a 40 ninfas de terceiro estágio do Triatoma infestans, por cepa, mantidas no insectário da Faculdade de Medicina do Triângulo Mineiro. As ninfas foram alimentadas sobre camundongos infectados com as diferentes cepas de Trypanosoma cruzi no pico máximo de parasitemia. Após o repasto sangüíneo, observou-se os volumes abdominais de cada triatomíneo, sendo descartados aqueles que não tinham feito uma boa alimentação.

Aos $30,60,90,120$ e 150 dias, doisobservadores examinaram as fezes de cada triatomíneo pelo 
Nirschl RA, Soares Júnior JM, Pirani EM, Franciscon JU, Ramirez LE. Susceptibilidade do Triatoma infestans a diferentes cepas de Trypanosoma cruzi isoladas de pacientes chagásico do Triângulo Mineiro. Revista da Sociedade Brasileira de Medicina Tropical 27:235-239, out-dez, 1994.

método da compressão abdominal e/ou dejeção espontânea e os barbeiros foram separados quanto ao resultado positivo ou negativo em recipientes devidamente rotulados; logo procedia-se a realimentação em camundongos sádios. $O$ anterior repetiu-se a cada leitura.

Para análise estatística, utilizou-se o teste de qui-quadrado $\left(\mathrm{X}^{2}\right)$ e o nível de significância adotado foi da propabilidade ser menor ou igual a 0,05 .

\section{RESULTADOS}

A Tabela 1 mostra a positividade do $T$. infestans às diferentes cepas de Trypanosoma cruzi. Observase que a positividade na primeira leitura variou de $61,1 \%$ (cepa E) a $42,6 \%$ (cepa B). Na segunda leitura, as porcentagens de positividade diminuiram, com excecão da cepa $H$ que teve sua postividade aumentada para 58,3\%. Na quarta leitura, os valores porcentuais diminuíram com relação à leitura anterior $e$, por último, na quinta leitura, a cepa $\mathrm{H}$ voltou a aumentar sua positividade enquanto que com as outras cepas isso não aconteceu. Não houve diferença estatisticamente significante entre as cepas em quaisquer leituras.

De um total de 182 triatomíneos vivos examinados após a primeira leitura, apenas $4(2,2 \%)$ se mantiveram positivos durante todas as leituras $\mathrm{e}$ $25(13,7 \%)$ permaneceram negativos. Contudo 113 $(62,08 \%)$ triatomíneos tiveram leituras ora positivas, ora negativas no decorrer de todo o estudo (resul tados não apresentados em tabelas).
A Tabela 2 apresenta o número e porcentagem de mortalidade de $T$. infestans através das cinco leituras. Observou-se um comportamento irregular para todas as cepas, isto é, a mortalidade variou ora aumentando ora diminuindo. Deve-se salientar que a mortalidade foi elevada (60\%). As cepas A e R na segunda leitura e $A$ e $G$ na terceira leitura apresentaram maior mortalidade em relação às demais cepas, o qual foi confirmado estatisticamente. Nas outras leituras, não foi observada diferença estatística significante.

$\mathrm{Na}$ Tabela 3, encontram-se as porcentagens acumulativas das diferentes cepas através das cinco leituras realizadas. Observa-se, em relação as cepas $A e G$, que apenas um triatomíneo tornou-se positivo após a $1^{\text {a }}$ leitura. Na cepa $E$, dois triatomíneos positivaram-se entre a segunda e terceira leitura. As cepas $B, H, R$ eW apresentam também um aumento de positividade entre a primeira e terceira leitura sendo de 7, 6, 3 e 3 respectivamente, apesar disso não houve diferença estatisticamente significante entre as leituras.

\section{DISCUSSÃO}

Perlowagora Szumlewicze Müller ${ }^{11}$ observaram que, quando o triatomínio infectado era deixado sem alimento por longos períodos, ocorria uma diminuição da multiplicação do parasita dentro do intestino do vetor levando a uma negativação do mesmo. Tendo em conta esse achado, em nosso trabalho os insetos foram realimentados num período

Tabela 1 - Número e porcentagem de positividade do Triatoma infestans às diferentes cepas de Trypanossoma cruzi através das cinco leituras.

\begin{tabular}{|c|c|c|c|c|c|c|c|c|c|c|}
\hline \multirow{3}{*}{ Cepas } & \multicolumn{10}{|c|}{ Leituras } \\
\hline & \multicolumn{2}{|c|}{$1^{a}$} & \multicolumn{2}{|c|}{$2^{a}$} & \multicolumn{2}{|c|}{$3^{a}$} & \multicolumn{2}{|c|}{$4^{a}$} & \multicolumn{2}{|c|}{$5^{a}$} \\
\hline & $n^{0}$ & $\%$ & $n^{0}$ & $\%$ & $\mathrm{n}^{0}$ & $\%$ & $n^{0}$ & $\%$ & $n^{0}$ & $\%$ \\
\hline E & $11 / 18$ & 61,1 & $6 / 17$ & 35,3 & $4 / 10$ & 40,0 & $2 / 9$ & 22,2 & $1 / 7$ & 14,3 \\
\hline A & $12 / 20$ & 60,0 & $4 / 11$ & 36,4 & $5 / 7$ & 71,4 & $4 / 6$ & 66,7 & $2 / 5$ & 40,0 \\
\hline H & $15 / 27$ & 55,6 & $14 / 24$ & 58,3 & $11 / 22$ & 50,0 & $5 / 18$ & 27,8 & $6 / 14$ & 42,9 \\
\hline W & $10 / 20$ & 50,0 & $5 / 18$ & 27,8 & $6 / 17$ & 35,3 & $3 / 11$ & 27,3 & $0 / 7$ & - \\
\hline $\mathrm{R}$ & $16 / 36$ & 44,4 & $6 / 26$ & 23,1 & $9 / 25$ & 36,0 & $7 / 22$ & 31,8 & $5 / 20$ & 25,0 \\
\hline G & $10 / 23$ & 43,5 & $7 / 19$ & 36,8 & $4 / 11$ & 36,4 & $2 / 6$ & 33,3 & $1 / 5$ & 20,0 \\
\hline B & $16 / 38$ & 42,6 & $11 / 32$ & 34,4 & $10 / 27$ & 37,0 & $7 / 25$ & 28,0 & $5 / 22$ & 22,7 \\
\hline $\mathrm{X}^{2}$ & \multicolumn{2}{|c|}{$\mathrm{p}=0,71$} & \multicolumn{2}{|c|}{$p=0,26$} & \multicolumn{2}{|c|}{$p=0,65$} & \multicolumn{2}{|c|}{$p=0,65$} & \multicolumn{2}{|c|}{$p=0,44$} \\
\hline
\end{tabular}


Nirschl RA, Soares Júnior JM, Pirani EM, Franciscon JU, Ramírez LE. Susceptibilidade do Triatoma infestans a diferentes cepas de Trypanosoma cruzi isoladas de pacientes chagásico do Triângulo Mineiro. Revista da Sociedade Brasileira de Medicina Tropical 27:235-239, out-dez, 1994.

Tabela 2 - Número e porcentagem de mortalidade do Triatoma infestans através das cinco leituras realizadas.

\begin{tabular}{|c|c|c|c|c|c|c|c|c|c|c|}
\hline \multirow{3}{*}{ Cepas } & \multicolumn{10}{|c|}{ Leituras } \\
\hline & \multicolumn{2}{|c|}{$1^{\mathrm{a}}$} & \multicolumn{2}{|c|}{$2^{\mathrm{a}}$} & \multicolumn{2}{|c|}{$3^{a}$} & \multicolumn{2}{|c|}{$4^{a}$} & \multicolumn{2}{|c|}{$5^{a}$} \\
\hline & $\mathrm{n}^{0}$ & $\%$ & $n^{0}$ & $\%$ & $n^{0}$ & $\%$ & $n^{0}$ & $\%$ & $\mathrm{n}^{0}$ & $\%$ \\
\hline G & $5 / 28$ & 17,9 & $4 / 23$ & 17,4 & $8 / 19$ & 42,1 & $5 / 11$ & 45,5 & $1 / 6$ & 16,7 \\
\hline W & $4 / 24$ & 16,7 & $2 / 20$ & 10,0 & $1 / 18$ & 5,6 & $6 / 17$ & 35,3 & $4 / 11$ & 36,4 \\
\hline $\mathrm{H}$ & $4 / 31$ & 12,9 & $3 / 27$ & 11,1 & $2 / 24$ & 8,3 & $4 / 22$ & 18,2 & $4 / 18$ & 22,2 \\
\hline $\mathrm{E}$ & $2 / 20$ & 10,0 & $1 / 18$ & 5,6 & $7 / 17$ & 41,0 & $1 / 10$ & 10,0 & $2 / 9$ & 22,2 \\
\hline B & $2 / 40$ & 5,0 & $6 / 38$ & 15,8 & $5 / 32$ & 15,6 & $2 / 27$ & 7,4 & $3 / 25$ & 12,0 \\
\hline $\mathbf{R}$ & $1 / 37$ & 2,7 & $10 / 36$ & 27,8 & $1 / 26$ & 3,8 & $3 / 25$ & 12,0 & $2 / 22$ & 9,8 \\
\hline A & $0 / 20$ & 0,0 & $9 / 20$ & 45,0 & $4 / 11$ & 36,4 & $1 / 7$ & 14,3 & $1 / 6$ & 16,7 \\
\hline$X^{2}$ & $p=$ & & $p=$ & & $p=c$ & & & & &, 57 \\
\hline
\end{tabular}

Tabela 3 - Número e porcentagem acumulativa de positividade das diferentes cepas através de 5 leituras.

\begin{tabular}{|c|c|c|c|c|c|c|c|c|c|c|c|c|c|c|}
\hline \multirow{3}{*}{$\begin{array}{l}\text { Leitu- } \\
\text { ras }\end{array}$} & \multicolumn{14}{|c|}{ Cepas } \\
\hline & \multicolumn{2}{|c|}{ A } & \multicolumn{2}{|c|}{$\mathrm{G}$} & \multicolumn{2}{|c|}{ E } & \multicolumn{2}{|c|}{ B } & \multicolumn{2}{|c|}{$\mathbf{H}$} & \multicolumn{2}{|c|}{$\mathbf{R}$} & \multicolumn{2}{|c|}{ W } \\
\hline & $n^{0}$ & $\%$ & $n^{0}$ & $\%$ & $n^{0}$ & $\%$ & $n^{0}$ & $\%$ & $\mathrm{n}^{0}$ & $\%$ & $n^{0}$ & $\%$ & $\mathrm{n}^{\circ}$ & $\%$ \\
\hline $1^{a}$ & $12 / 20$ & 60,0 & $10 / 28$ & 35,7 & $11 / 20$ & 55,0 & $16 / 40$ & 40,0 & $15 / 31$ & 48,4 & $16 / 37$ & 43,2 & $10 / 24$ & 41,7 \\
\hline $2^{a}$ & $13 / 20$ & 65,0 & $11 / 28$ & 39,3 & $11 / 20$ & 55,0 & $20 / 40$ & 50,0 & $20 / 31$ & 64,5 & $17 / 37$ & 45,9 & $12 / 24$ & 50,0 \\
\hline $3^{a}$ & $13 / 20$ & 65,0 & $11 / 28$ & 39,3 & $13 / 20$ & 65,0 & $23 / 40$ & 57,5 & $21 / 31$ & 67,7 & $19 / 37$ & 51,3 & $13 / 24$ & 54,2 \\
\hline $4^{a}$ & $13 / 20$ & 65,0 & $11 / 28$ & 39,3 & $13 / 20$ & 65,0 & $23 / 40$ & 57,5 & $21 / 31$ & 67,7 & $19 / 37$ & 51,3 & $13 / 24$ & 54,2 \\
\hline $5^{a}$ & $13 / 20$ & 65,0 & $11 / 28$ & 39,3 & $13 / 20$ & 65,0 & $23 / 40$ & 57,5 & $21 / 31$ & 67,7 & $19 / 37$ & 51,3 & $13 / 24$ & 54,2 \\
\hline$X^{2}$ & & & & & $\mathrm{p}=\mathrm{c}$ & & $p=c$ & & $p=$ & & $p=$ & & $\mathrm{p}=\mathrm{c}$ &, 89 \\
\hline
\end{tabular}

de 20 a 30 dias após a alimentação anterior em camundongos sadios, evitando-se alimentar sobre animais diferentes com o fim de eliminar outros fatores que pudessem influenciar na susceptibilidade do vetor ${ }^{14}$.

Todos os triatomíneos, nesse trabalho, foram examinados a cada leitura pelos métodos da compressão abdominal e/ou dejeção espontânea. Segundo Bronfen e Alvarenga ${ }^{4}$, existem flutuações na positividade do $T$. infestans, especialmente pelo método da compressão abdominal devido à baixa densidade de flagelados o que implica uma grande dificuldade de visualização do parasita, podendo levar a um resultado falso negativo. Na tentativa de corrigir esse erro, as fezes obtidas decada triatomíneo eram examinadas minuciosamente por 2 observadores.

Utilizamos, em nosso estudo, o T. infestants que é um vetor adaptado à região do Triângulo Mineiro e domiciliado, e cepas de Trypanosoma cruzi isoladas da mesma área. Perlowagora Szumlewicz e Müller ${ }^{10}$ observaram que a infecção de vetores domiciliados era moderada e passageira $e$, nos vetores silvestres, era maciça e persistente $e$ concluíram que a domiciliação leva a uma mudança na interação vetor-parasita sem razão definida. Em outro estudo feito pelos mesmos autores ${ }^{11}$, concluíram que isso poderia estar associado a uma mutação adaptativa do vetor local com o parasita do mesmo local.

As cepas utilizadas na experiência são caracterizadas pelo predomínio de formas largas e 
Nirschl RA, Soares Júnior JM, Pirani EM, Franciscon JU, Ramirez LE. Susceptibilidade do Triatoma infestans a diferentes cepas de Trypanosoma cruzi isoladas de pacientes chagásico do Triangulo Mineiro. Revista da Sociedade Brasileira de Medicina Tropical 27:235-239, out-dez, 1994.

por serem de baixa virulência e patogenicidade em camundongos, a exceção das cepas B e H que são de elevada virulência, baixa patogenicidade e tem predomínio de formas delgadas no início da infecção, porém não se encaixando em os parâmetros propostos por Andrade ${ }^{1}$. Vários estudos ${ }^{3} 12$ têm demonstrado uma maior susceptividade do $T$. infestans pelas cepas com predomínio de formas largas, entretanto, isso não foi observado nesse trabalho, já que todas as cepas apresentaram um comportamento semelhante no vetor.

Nossos resultados demonstraram uma positividade de $62,08 \%$ e uma mortalidade de $60 \%$, no decorrer dos 150 dias da experiência, observandose que apenas $4(2,2 \%)$ triatomíneos permaneceram positivos e $25(13,9 \%)$ negativos , por todo o tempo.

Perlowagora e Müller ${ }^{10}$ alimentando T. infestans em camundongos infectados com $T$. cruzi, na fase aguda, obtiveram uma positividade de $51 \%$. Os mesmos autores ${ }^{11}$ encontraram uma positividade semelhante $(53,7 \%)$ quando alimentaram $T$. infestans sobre cobaias infectadas e realimentaram com sangue de galinha. Sherlock ${ }^{14}$, alimentando $R$. prolixus, que é um vetor altamente domiciliado na Venezuela e Colombia ${ }^{8}$, obteve uma positividade de $67 \%$. Schenone e $\operatorname{cols}^{13}$ realizaram xenodiagnósticos com $T$. infestans em uma paciente com doença de Chagas crônica e parasitemia persistente, observaram que só $40 \%$ dos triatomineos empregados foram infectados.

Tolezano e cols ${ }^{16} 17$ demonstraram que triatomíneos não manipulados têm maior sobrevivência e capacidade de sugar que os manipulados. Nossos dados demonstram que se as mortes fossem causadas só pela manipulação, não teria ocorrido nenhuma mortalidade ou essa seria mínima no intervalo entre a alimentação inicial em camundongos contaminados e a primeira leitura; porém, Schenone e cols ${ }^{13}$ observaram que a mortalidade não estava relacionada com a positividade do triatomíneo devido ao fato que a mortalidade em seu trabalho foi maior para o grupo não infectado. Os mesmos autores demonstraram que a maioria dos insetos apresentavam leituras ora positivas, ora negativas, o qual também foi observado em nosso trabalho.

Em resumo, pode-se concluir que, para qualquer cepa de $T$. cruzi da região do Triângulo Mineiro empregada, a susceptibilidade do Triatomainfestans foi baixa e que as mesmas conservaram suas características com respeito às observadas em outras regiōes do país. Outro fato que colabora com os achados do nosso estudo são os obtidos de xenodiagnósticos realizados na Faculdade de Medicina do Triângulo Mineiro, onde num intervalo de 10 anos (1983-1993), utilizando-se esses triatomíneos, foram feitos 613 xenodiagnósticos em pacientes provenientes de diferentes regiões do Triângulo Mineiro, comprovadamente chagásicos pelos testes de imunofluorescência indireta, hemaglutinação indireta e fixação de complemento, encontrando-se uma positividade de 149 (24,3\%) não muito diferente da obtida em outros laboratórios com o mesmo triatomíneo.

\section{SUMMARY}

This data evaluated the susceptibility of $\mathrm{T}$. infestans to seven different strains of $\mathrm{T}$. cruzi from chagasic patients of the Triângulo Mineiro, isolated and adapted in Swiss mice. A total of 200 bugs were fed in mice in acute phase injecled with the different strains of $\mathrm{T}$. cruzi and at each 30 days, during 5 months, their feces were obtained by abdominal compression or spontaneous dejection. The overall positivity of $\mathrm{T}$. infestans infection with these different strains of $\mathrm{T}$. cruzi was $62.08 \%$. There was a high mortality rate $(60 \%)$ during the examination period. We conclude that $\mathrm{T}$. infestans has a low susceptibility to these strains. This could explain the low rate of positivity in the xenodiagnosis in chagasic chronic patients of the Triangulo Mineiro.

Key-words: Triatoma infestans' susceptibility. Trypanosoma cruzi. Xenodiagnosis.

\section{AGRADECIMENTOS}

Agradecemos à senhorita Aparecida Correa pela assistência técnica.

\section{REFERÊNCIAS BIBLIOGRÁFICAS}

1. Andrade SG. Caracterização de Cepas do Trypanosoma cruzi isoladas no Recôncavo Bahiano (contribuição ao estudo de patologia geral da doença de Chagas em nosso meio). Tese de Professor Assistente, Faculdade de Medicina da Universidade Federal da Bahia, Salvador, BA, Brasil, 1973.

2. Barreto AC, Marsden PD, Cuba CC, Alvarenga NJ. Estudo preliminar de Dipetalogaster maximus na 
Nirschl RA, Soares Jtinior JM, Pirani EM, Franciscon JU, Ramírez LE. Susceptibilidade do Triatome iniestans a diferentes cepas de Trypanosoma cruzi isoladas de pacientes chagásico do Triângulo Mineiro. Revista da Sociedade Brasileira de Medicina Tropical 27:235-239, out-dez, 1994.

técnica do xenodiagnóstico em forma crônica de Doença de Chagas. Revista do Instituto de Medicina Tropical 20:183-189, 1978.

3. Bronfen E. Susceptibility of $T$. infestans and $P$. megistus to infection by $\mathrm{X} T$, cruzi strain. In: Resumos do Congresso Internacional sobre Doença de Chagas, Rio de Janeiro, p. 38-39, 1979.

4. Bronfen $\mathbf{E}$, Alvarenga NJ. O xenodiagnóstico para avaliar o nível de parasitemia do paciente chagásico crônico. Revista da Sociedade Brasileira de Medicina Tropical 24:37-42, 1991.

5. Camargo ME, Takeda GKF. Diagnóstico de Laboratório. In: Andrade Z, Brener Z (eds) Trypanosoma cruzi e Doença de Chagas. GuanabaraKoogan, Rio de Janeiro p. 175-198, 1979.

6. Dias JCP. Mecanismos de transmissão. In: Andrade, Z; Brener, $Z$ (eds) Trypanosoma cruzi e Doença de Chagas. Guanabara-Koogan, Rio de Janeiro, p. 152-174, 1979.

7. Maudlin 1. Inheritance of susceptibility to $T$. cruzi infection in Rhodnius prolixus. Nature 262:214215,1976 .

8. Organización Mundial de la Salud. Control de la Enfermedad de Chagas. Informe de un comité de Expertos de la OMS. Série de Informes tecnicos $\mathrm{N}^{\circ}$ 811. Genebra, 1991.

9. Perlowagora-Szumlewicz A, Müller CA. Experiments in a search for an insect model for xenodiagnosis of chronic Chagas' disease. In: Resumos do Congresso Internacional sobre doença de Chagas, Rio de Janeiro p. E11-E16, 1979.

10. Perlowagora-Szumlewicz A, Müller CA. Studies in search of a suitable experimental insect model for diagnosis of hosts with Chagas' desease. 1Comparative xenodiagnosis of hosts with nine triatomine species of animals with acute infections by Trypanosoma cruzi. Memórias do Instituto Oswaldo Cruz 77:37-53, 1982.

11. Perlowagora-Szumlewicz A, Müller CA. Studies in search of a suitable experimental insect model for xenodiagnosis of hosts with Chagas' disease. 2 -
Attempts to upgrade the reliability and the efficacy of xenodiagnosis in chronic Chagas' disease. Memórias do Instituto Oswaldo Cruz 82: 259-272, 1987.

12. Ruas LAF. Contribuição para o estudo do xenodiagnóstico. Aspecto da relação entre amostras do $T$. cruzi e espécies de triatomíneos. Tese de Mestrado, Faculdade de Medicina de Ribeirão Preto, Universidade de São Paulo, Ribeirão Preto, 1977.

13. Schenone H, Raja M, Rajas A, Concha L. Positividad diurna y noturna del xenodiagnóstico en un paciente con infeccion chagásica crónica de parasitemia permanente, Boletin Chileno de Parasitologia 9: 6366, 1977.

14. Sherlock IA. Parasitemia constante durante 24 horas consecutivas na infeç̧ão experimental pelo Trypanosoma cruzi. Revista da Sociedade de Medicina Tropical 17:137-144, 1984

15. Sherlock IA, Almeida SP. Diferença de susceptibilidade à infecção com Trypanosoma cruzi entre espécies de triatomíneos alimentados em cão, tatue camundongos infectados. Revista da Sociedade Brasileira de Medicina Tropical 7:87-98, 1973.

16. Tolezano JE, Chiffi PP, Araujo MFL, Valentin AM, Ribeiro SS. Variáveis relacionadas ao desenvolvimento de $T$. infesians Klug. $1834 \mathrm{em}$ condições de laboratório. 1-Relação entre o repasto sangüíneo e o desenvolvimento. Revista do Instituto Adolfo Lutz 44:73-79, 1984.

17. Tolezano JE, Chiffi, PP, Araujo MFL, Valentin AM, Ribeiro SS. Variáveis relacionadas ao desenvolvimento de $T$. infestans Klug. $1834 \mathrm{em}$ condições de laboratório. 2- Efeito da manipulação na sobrevivência e fertilidade. Revista do Instituto Adolfo Lutz 44:81-86, 1984.

18. Zeledon R. Effects of triatomine behaviour on trypanosome transmission. In: American Trypanosomiasis Research. Proceedings of an International Symposium. Pan American Health Organization, Belo Horizonte, MG, p. 326 - 329, 1975. 